\title{
Spin-vortex Superconductivity
}

\author{
Hiroyasu Koizumi
}

Received: 3 February 2011 / Accepted: 9 March 2011 / Published online: 1 April 2011

(C) The Author(s) 2011. This article is published with open access at Springerlink.com

\begin{abstract}
We present a theory of superconductivity based on the theoretical prediction that a macroscopic persistent current is generated by spin-vortices. It explains the origin of the phase variable $\theta$ that is canonical conjugate to the superfluid density as a Berry phase arising from the spin-vortex formation. This superconductivity does not require Cooperpairs as charge carriers, thus, is not directly related to the standard theory based on the BCS one; however, it exhibits the flux quantization in the unit $\Phi_{0}=h c / 2|e|$, where $h$ is Planck's constant, $c$ the speed of light, and $e$ the electron charge; and the AC Josephson frequency, $f_{\mathrm{J}}=2|e| V / h$, where $V$ is the voltage of the battery connected to the superconductor-insulator-superconductor junction. In due course, it is found that the standard derivation of the AC Josephson frequency misses a term arising from the flow of particles through the leads connected to the junction. If this contribution is included, the observed $f_{\mathrm{J}}$ indicates that the phase $\theta$ is a variable conjugate to the number density of charge $e$ carriers instead of the currently accepted charge $2 e$ carriers. We propose an experiment that discriminates whether it is $e$ or $2 e$. If the above claim is verified, it means that the BCS theory cannot predict whether a particular compound is a superconductor or not since it does not explain the origin of $\theta$. A connection between the present mechanism and the BCS mechanism is discussed; the fact that the BCS theory gives an excellent estimate of $T_{\mathrm{c}}$ is attributed to the fact that it predicts the temperature at which spin-vortices become long-lived due to the energy gap formation; since the stabilization by the electron-pair formation is compatible with the present mechanism, asymmetries observed in the
\end{abstract}

H. Koizumi $(\bowtie)$

Institute of Materials Science, University of Tsukuba, Tsukuba, Ibaraki 305-8573, Japan

e-mail: koizumi@ims.tsukuba.ac.jp even and odd number of electron systems are preserved. The most notable difference is that the persistent current generation is formulated in a strictly particle-number-conserving manner. Thus, it does not violate the superselection rule for the total charge.

Keywords Phase of the superconducting order parameter . Spin-vortices · Berry phase - Josephson effect - Cuprates . Loop currents · Magnetic excitations

\section{Introduction}

In 1911, the superconducting state of electrons was discovered in pure mercury [1]; electric charge was transferred between two end points of a sample without a voltage drop.

This superfluid phenomenon of charged particles has been explained as a consequence of an energy gap formation by the electron-pair (Cooper-pair) condensation [2]. Since the superfluidity of ${ }^{4} \mathrm{He}$ is strongly connected to BoseEinstein condensation (BEC) [3, 4], superconductivity is often regarded as the consequence of the BEC of Cooper-pairs, where Cooper-pairs are regarded as bosons.

The supercurrent is believed to be proportional to the gradient of a phase variable " $\theta$ " that is canonical conjugate to the density of the particles $\rho$ [5]; actually, this phase $\theta$ is the key ingredient that causes many phenomena associated with superconductivity.

Although $\theta$ is crucially important, its origin is not settled, yet; there are more than one theories for its origin. The most popular one is the one by P.W. Anderson [6]. He reached his conclusion through the prediction and observation of a DC current flow through a weak-linked superconductors without a voltage difference, and an AC current flow with the nonzero voltage difference by B.D. Josephson [7, 8]. He argues 
that the gauge symmetry is broken in the BEC system in the sense that the mean value of the particle field operator $\hat{\psi}$ has a macroscopic value;

$\langle\hat{\psi}(\mathbf{r}, t)\rangle=\rho^{1 / 2}(\mathbf{r}, t) e^{-i \theta(\mathbf{r}, t)}$.

In this treatment, $\theta$ arises as a consequence of the gauge symmetry breaking.

The currently accepted theory of superconductivity is the one based on the BCS theory which provides a way to calculate $T_{\mathrm{c}}$, and explains many properties associated with an energy gap formation due to the Cooper-pair condensation. In this theory, the superconducting wave function is given as a linear combination of different particle-number states, thus, the field operator has non-zero mean values in accordance with (1).

The supercurrent is a flow of Cooper-pairs in the BCS theory; thus, $\hat{\psi}$ is the field operator for Cooper-pairs. The carrier charge $q$ of the supercurrent should be $q=2 e$ since the charge on a Cooper-pair is $2 e$, where $e$ is the electron charge. The observed flux quantization unit [9]

$\Phi_{0}=\frac{h c}{2|e|}$,

where $h$ is Planck's constant and $c$ the speed of light; and the AC Josephson frequency [10],

$f_{\mathrm{J}}=\frac{2|e| V}{h}$,

where $V$ is the voltage of the battery connected to the Josephson (or superconductor-insulator-superconductor (SIS)) junction are believed to be the manifestation of $q=2 e$.

Although the above gauge symmetry breaking origin of $\theta$ is very popular, it is not universally accepted; there is a serious claim by A.J. Leggett that observed phenomena are equally explainable without the gauge symmetry breaking if the BEC occurs [11]. In this view, the gauge symmetry breaking is just a calculational tool, and care must be taken for any properties that are calculated with violating the conservation of the particle number. It is also worth noting that Anderson's view violates the superselection rule for the total charge [12-14].

Actually, there is a loose end of the fundamental level in the standard theory of superconductivity; even the existence of the phase variable $\theta$ is granted, there is no convincing explanation for the thermodynamic stability (or quasistability) of the current proportional to $\nabla \theta$ [14]; i.e., $\nabla \theta=0$ should be satisfied in the ground state, thus, any current carrying state with $\nabla \theta \neq 0$ should be relaxed to the currentless state. However, the standard theory does not provide a convincing explanation for the long-lived $\nabla \theta \neq 0$; this also means that we really do not know the true connection between the BEC and superfluidity. Practically, persistent current related phenomena are calculated using the GinzburgLandau phenomenological equation [5] assuming that the stability of $\nabla \theta$ is something granted.

Meanwhile, a quite different theory for the persistent current generation has been put forward [15-17]. In this theory the phase $\theta$ arises as a Berry phase due to the spin-vortex formation; loop currents are predicted to be induced as a Berry phase effect if spin-vortices are present. The stability of $\nabla \theta \neq 0$ is explained as the stability of the spin-vortices. This theory also yields the flux quantization in the unit $\Phi_{0}$ as in the standard superconductivity theory.

In the above persistent current, a multi-valued function $\chi$ appears; it is related to $\theta$ as $\theta=-\chi / 2$, and satisfies

$\nabla^{2} \chi=0$

and the current is given roughly proportional to $\nabla \chi$ [15]. Actually, such a function was speculated to exist in superconductors by F. London, and called, the "superpotential" [18]. It is worth noting that the existence of an electric current proportional to $\nabla \chi$ invalidates the usual decomposition of electric current into the transverse and longitudinal components [19]. Since $\nabla \chi$ is a harmonic vector, it satisfies both the transverse condition $\nabla \cdot \nabla \chi=0$ and the longitudinal condition $\nabla \times \nabla \chi=0$, thus, $\nabla \chi$ will give rise to extra transverse and longitudinal contributions.

The above-mentioned new development suggests that a theory of superconductivity may be constructed in a quite different manner; i.e., the required phase $\theta$ in superconductivity arises without referring to the BEC or the gauge symmetry breaking.

At this point, it is worth noting that the standard theory of superconductivity is not powerful in predicting whether a particular compound is a superconductor or not. This is usually excused by the difficulty in obtaining normal state properties from which $T_{\mathrm{c}}$ is calculated. However, it has been argued that the normal state properties that are important in predicting $T_{\mathrm{c}}$ is not statistically correlated with whether a particular compound is a superconductor or not [20]. It is also observed in $\mathrm{La}_{1.875} \mathrm{Ba}_{0.125} \mathrm{CuO}_{4}$ that although an energy gap that appears to be due to the Cooper-pair condensation exists, it fails to be superconducting [21]. The above two facts may indicate that the origin of $\theta$ may be different from the Cooper-pair condensation, although the temperature for it corresponds to $T_{\mathrm{c}}$. It is also noteworthy that evidence for stable loop currents is observed in the cuprate by the polar Kerr-effect measurement [22, 23] and the recent neutron scattering experiments [24]; especially, the latter have observed magnetic excitations peaked at $\mathbf{q}=0$, suggesting the existence of a current of a harmonic vector.

In the present work, we propose the Berry phase origin for $\theta$. The most significant difference is that $\theta$ is a phase 
variable canonical conjugate to the number density of charge $e$ (not $2 e$ ) carriers. It is also noteworthy that the present origin does not violate the superselection rule for the total charge. We expect that the rigidity of $\theta$ [25] sets in due to the energy gap formation by the Cooper-pair condensation at $T_{\mathrm{c}}$, thus, the fact that the BCS theory gives an excellent estimate of $T_{\mathrm{c}}$ is explained. Since the stabilization by the electron-pair formation is compatible with the present mechanism, asymmetries observed in the even and odd number of electron systems are preserved.

Let us describe the organization of the present work. In Sect. 2, we first discuss the occurrence of the Josephson effect in a rather general manner. We put forward a new definition of the superfluid system. Our definition is "the system equipped with $\theta$ that is canonical conjugate to $\rho$, and provides a long-lived current proportional to $\nabla \theta$ ". We will demonstrate that the above definition and gauge invariance requirements gives rise to the Josephson effect.

In the process of re-deriving the AC Josephson frequency, it is found that the conventional derivation misses a contribution from the flow-in and -out of particles through the leads connected to the Josephson junction. If this contribution is included, the observed AC Josephson frequency indicates that the charge on the tunneling particle is $e$, not $2 e$, or the phase $\theta$ is conjugate to the number density of charge $e$ carriers. This finding upsets the standard theory; thus, it should be verified experimentally. For this purpose, we propose an experiment that discriminates whether the tunneling charge across the Josephson junction is in the unit of $e$ or $2 e$. Note here that the above statement does not exclude the apparently pairwise tunneling; if experiments are performed in the condition where the paring stability is significant compared to other energies such as the thermal energy $k_{\mathrm{B}} T\left(k_{\mathrm{B}}\right.$ is the Boltzmann constant) or the Coulomb energy, asymmetry in even and odd particle-number states will be observed.

In Sect. 3, we derive the phase $\theta$ from a model contains stable spin-vortices. Actually, $\theta$ is given as a Berry phase induced by the spin-vortices. This Berry phase arises from the twisting of two basis functions, thus, mathematically equivalent to the one first found by Longuet-Higgins et al. in the context of the dynamical Jahn-Teller effect [34, 35]; the two basis functions here are, however, the two spin states of an electron, while they are those of a doubly degenerate $E$ symmetry state in the Jahn-Teller case. When an energy gap for electronic excitations is formed, the stability in $\nabla \theta$ is realized.

In Sect. 4, we argue that stable spin-vortices are formed in the effectively half-filled Mott insulator (EHFMI) state, and a stable $\nabla \theta$ is achieved $[15,26]$. Here, the half-filled insulating state is approximated by a Slater determinant, thus, the EHFMI state may be also called "the effectively half-filled Slater state (EHFSS)" when we emphasize the current generation aspect. In the EHFSS, the lower band is filled with extended single-particle states [27]; usually, a filled band means an insulator, however, thanks to the fictitious magnetic field generated by spin-vortices, the EHFSS can be a current carrying state.

In Sect. 5, the oscillation of the maximum zero-voltage current with varying an external magnetic field in a circuit with two identical SIS junctions (the SQUID structure) is examined. We show that the present theory explains observed oscillation patterns [28]. We also demonstrate that the "0-SQUID (or edge-junction)" and " $\pi$-SQUID (or cornerjunction)" patterns [29] that are usually attributed to the dwave symmetry of the order parameter, are shown to arise from evenness or oddness of the winding number of the Berry phase.

Lastly in Sect. 6, we will conclude this work by discussing a possible relation between the present theory and the BCS theory. The relevance of the present theory to the cuprate superconductivity is also discussed, where it is suggested that the BCS gap formation mechanism is necessary for superconductivity in the surface region where the small polaron formation required for the EHFMI state (or EHFSS) is not fulfilled.

\section{A Definition of Superconductivity}

P.W. Anderson has taken that a system with the order parameter $\Psi$ defined by

$\Psi=\langle\hat{\psi}(\mathbf{r}, t)\rangle=\rho^{1 / 2}(\mathbf{r}, t) e^{-i \theta(\mathbf{r}, t)}$,

as the definition of the superfluid system [6], where $\rho$ is the particle density and $\theta$ is a phase variable.

The variables $\theta$ and $\rho$ are canonical conjugate; thus, the following basic equations of motion are obtained;

$\dot{\rho}=\frac{1}{\hbar} \frac{\delta H}{\delta \theta}$,

$\dot{\theta}=-\frac{1}{\hbar} \frac{\delta H}{\delta \rho}$,

where $H$ is an energy functional that depends on $\rho$ and $\theta$.

In deriving the above equations, Anderson has used the fact that the BCS state vector is a linear combination of state vectors for different numbers of Cooper-pairs, and assumed the phases among them are meaningful, i.e., the superselection rule for the charge [12] is violated. Since the charge on a Cooper-pair is $2 e$, the phase $\theta$ is conjugate to the number density for $2 e$ charge carriers.

Later, A.J. Leggett has obtained the same equations by considering wave functions for the Bose-Einstein condensate $[11,14]$. In his treatment, the Cooper-pair is treated as a boson; thus, $\theta$ is the phase variable conjugate to the number density for $2 e$ charge carriers as in the Anderson's treatment. 


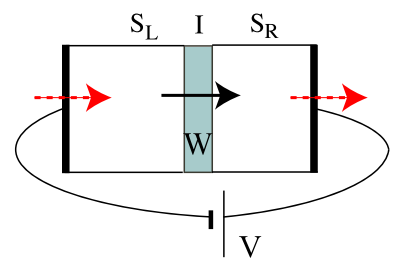

Fig. 1 A Josephson junction contacted with a battery of a voltage $V$. An electric field appears inside the insulator (denoted as "I" in the figure) since the junction is a capacitor in the zeroth order approximation. The voltage across the insulator is denoted as $W$. The tunneling of a charged particle over the insulator (solid-line arrow) is accompanied by the flow of charged particles (dotted-line arrows) in and out of the junction

However, the conservation of the number of particles is preserved.

In the following, we go in the other direction; we take "the existence of the phase variable $\theta$ that is canonical conjugate to $\rho$, and that gives rise to a long-lived current depends on $\nabla \theta$ " as the definition of the superfluid system. We will derive the AC Josephson frequency starting from (6) and (7), and gauge invariance.

Since $\theta$ is a phase variable, the energy functional should depend on $\theta$ through $\nabla \theta$. Then, (6) describes merely the equation of continuity,

$\dot{\rho}+\nabla \cdot \mathbf{j}=0$,

where the current density $\mathbf{j}$ is given by

$\mathbf{j}=\frac{1}{\hbar} \frac{\delta H}{\delta \nabla \theta}$.

For the supercurrent dynamics, (7) is the most important one.

Let us consider an superconductor-insulator-superconductor (SIS) junction or a Josephson junction. The two superconductors are denoted as $\mathrm{S}_{L}$ and $\mathrm{S}_{R}$; they are contacted with a battery of a voltage $V$ as is seen in Fig. 1. Note that the situation depicted in Fig. 1 is a very simplified one; in reality other elements of circuits exist; what is important here is that an energy difference of $|q| V$ exists between the particle that enters and another one that exits ( $q$ is the charge on the particle), and an electric field appears in the insulator to maintain the chemical potential difference between two superconductors.

For a while, we consider the case in which the voltage of the battery is zero, and ignore the electromagnetic interaction.

Equations (6) and (7) indicate that $\rho$ and $\nabla \theta$ are optimized in stationary states. We assume that the $\nabla \theta$ is optimized in each superconductor; note, however, that this dose not mean that current does not exist in each of them; a circular current may exist in each given by (9).
Since $\theta$ itself is arbitrary, the optimized $\theta$ in $\mathrm{S}_{L}$ and $\mathrm{S}_{R}$ are written as

$\theta(\mathbf{r})+\theta_{L}$

and

$\theta(\mathbf{r})+\theta_{R}$,

respectively, where $\theta_{L}$ and $\theta_{R}$ do not depend on the position.

Let us assume that the weak link is so weak that the optimized $\nabla \theta$ in $S_{L}$ and $S_{R}$ are not affected by it; however, $\theta_{R}-\theta_{L}$ is. In this case, we can treat $\theta_{R}$ and $\theta_{L}$ as timedependent variables that represent the time-dependence of $\theta$ in $\mathrm{S}_{R}$ and $\mathrm{S}_{L}$, respectively. Then, conjugate variables for $\theta_{R}$ and $\theta_{L}$ become the number of particles in $\mathrm{S}_{R}$ and $\mathrm{S}_{L}, N_{R}$ and $N_{L}$, respectively.

From (6) and (7), the equations for $N_{R}$ and $\theta_{R}$ are obtained as

$\dot{N}_{R}=\frac{1}{\hbar} \frac{\partial H}{\partial \theta_{R}}$

$\dot{\theta}_{R}=-\frac{1}{\hbar} \frac{\partial H}{\partial N_{R}}$,

and those for $N_{L}$ and $\theta_{L}$ as

$\dot{N}_{L}=\frac{1}{\hbar} \frac{\partial H}{\partial \theta_{L}}$,

$\dot{\theta}_{L}=-\frac{1}{\hbar} \frac{\partial H}{\partial N_{L}}$.

The quantum mechanical version of them is given by

$\dot{\hat{N}}_{A}=\frac{i}{\hbar}\left[\hat{H}, \hat{N}_{A}\right]$,

$\dot{\hat{\theta}}_{A}=\frac{i}{\hbar}\left[\hat{H}, \hat{\theta}_{A}\right]$,

where $A$ is either $R$ or $L ; \hat{H}, \hat{N}_{A}$, and $\hat{\theta}_{A}$ are operatorcorrespondences for $H, N_{A}$, and $\theta_{A}$, respectively.

Since $\hat{N}_{R}$, and $\hat{\theta}_{R}$ (and also $\hat{N}_{L}$, and $\hat{\theta}_{L}$ ) are canonical conjugate operators, the commutation relation of them is given by

$\left[\hat{N}_{A}, \hat{\theta}_{B}\right]=-i \delta_{A B}$,

where $A$ and $B$ are either $L$ or $R$.

By introducing the following operators:

$\hat{C}_{A}=e^{-i \theta_{A}} \hat{N}_{A}^{1 / 2}$

and

$\hat{C}_{A}^{\dagger}=\hat{N}_{A}^{1 / 2} e^{i \theta_{A}}$, 
the commutation relation in (18) becomes

$\left[\hat{C}_{A}, \hat{C}_{B}^{\dagger}\right]=\delta_{A B}$

this shows that $\hat{C}_{R}$ and $\hat{C}_{R}^{\dagger}\left(\hat{C}_{L}\right.$ and $\left.\hat{C}_{L}^{\dagger}\right)$ are the annihilation and creation operators for particles in $S_{R}\left(S_{L}\right)$. Note that the commutation relations are those for bosons, although the particles may be fermions; this is allowable since we consider the case $N_{L}, N_{R} \gg 1$.

Now, construct the operator for the transfer of particles between $\mathrm{S}_{R}$ and $\mathrm{S}_{L}$; the current between them is calculated with it. We use

$\hat{K}_{J}=t_{J} \hat{C}_{R}^{\dagger} \hat{C}_{L}+t_{J} \hat{C}_{L}^{\dagger} \hat{C}_{R}$

where $t_{J}$ is a hopping matrix element between $\mathrm{S}_{L}$ and $\mathrm{S}_{R}$.

Let us employ a semiclassical approximation in which $\hat{\theta}_{R}, \hat{\theta}_{L}, \hat{N}_{R}, \hat{N}_{L}$ are replaced by their mean values $\theta_{R}, \theta_{L}$, $N_{R}$, and $N_{L}$, respectively. Then, the semiclassical version of $\hat{K}_{J}$ is given by

$K_{J}=E_{J} \cos \left(\theta_{R}-\theta_{L}\right)$,

where

$E_{J}=2 t_{J}\left(N_{R} N_{L}\right)^{1 / 2}$

Using (12) and (23), the electric current from $S_{L}$ to $S_{R}$ is calculated as

$J=\frac{q E_{J}}{\hbar} \sin \left(\theta_{R}-\theta_{L}\right)$.

The derivative of the phase difference $\theta_{R}-\theta_{L}$ is calculated using (13) and (15);

$\dot{\theta}_{R}-\dot{\theta}_{L}=-\frac{1}{\hbar} \frac{\partial H}{\partial N_{R}}+\frac{1}{\hbar} \frac{\partial H}{\partial N_{L}}$.

If the chemical potential difference between $S_{R}$ and $S_{L}$ is zero, the phase difference is obtained as

$\theta_{R}-\theta_{L}=C$

where $C$ is a constant. This shows that even if the chemical potential difference (or voltage drop) is zero, a non-zero current

$J=\frac{q}{\hbar} E_{J} \sin C$

can flow. The phase difference is long-lived by assumption; therefore, the above $J$ describes a supercurrent.

If the particles are charged, they interact with the electromagnetic field. Then, the Hamiltonian $H$ contains the Coulomb term

$q \int d^{3} r \varphi \rho$ where $q$ is the charge on the particle and $\varphi$ is the electrostatic potential.

Then, the basic equations of motion given in (6) and (7) are modified as

$\dot{\rho}=\frac{1}{\hbar} \frac{\delta \bar{H}}{\delta \theta}$,

$\dot{\theta}=-\frac{q}{\hbar} \varphi-\frac{1}{\hbar} \frac{\delta \bar{H}}{\delta \rho}$,

where the gauge invariant energy functional $\bar{H}$ is defined as

$\bar{H}=H-q \int d^{3} r \varphi \rho$.

Equation (31) indicates that the sum

$\dot{\theta}+\frac{q}{\hbar} \varphi$

is gauge invariant since $\delta \bar{H} / \delta \rho$ is gauge invariant. This means that the spatial counterpart of it,

$\nabla \theta-\frac{q}{\hbar c} \mathbf{A}$

is also gauge invariant.

Therefore, the energy functional $\bar{H}$ should be a functional of the gauge invariant combination $\nabla \theta-\frac{q}{\hbar c} \mathbf{A}$, instead of $\nabla \theta$. From this fact, we can obtain the familiar expression for the electric current density formula,

$q \mathbf{j}=\frac{q}{\hbar} \frac{\delta \bar{H}}{\delta \nabla \theta}=-c \frac{\delta \bar{H}}{\delta \mathbf{A}}$

By taking into account the gauge invariance, (23) and (25) should be modified as

$K_{J}=E_{J} \cos \phi$

and

$J=\frac{q}{\hbar} E_{J} \sin \phi$

respectively, where

$\phi=\theta_{R}-\theta_{L}-\int_{L}^{R} \frac{q}{\hbar c} \mathbf{A} \cdot d \mathbf{r}$

In the following we consider the case where the magnetic field is absent; then, we may take $\mathbf{A}=0$, and (38) becomes

$\phi=\theta_{R}-\theta_{L}$.

The system is a closed-circuit with including the leads and the battery. When calculating the time-derivative of $\phi$, we need to take into account the tunneling through the insulator part of the junction, and the flow-in and -out of the 
particles through the leads. In order to clarify the inclusion of the two types of particle transfer processes, we express the Hamiltonian as

$H=H_{\mathrm{LIR}}+H_{\mathrm{LBR}}+H_{\mathrm{r}}$

where $H_{\mathrm{LIR}}$ is the term for the particle transfer through the insulator, and $H_{\mathrm{LBR}}$ is that through the leads; $H_{\mathrm{r}}$ expresses the remaining term.

Using (40), (26) is written as

$$
\begin{aligned}
\dot{\phi} & =\dot{\theta}_{R}-\dot{\theta}_{L} \\
& =\frac{1}{\hbar}\left(\frac{\partial H_{\mathrm{LIR}}}{\partial N_{L}}-\frac{\partial H_{\mathrm{LIR}}}{\partial N_{R}}+\frac{\partial H_{\mathrm{LBR}}}{\partial N_{L}}-\frac{\partial H_{\mathrm{LBR}}}{\partial N_{R}}\right) ;
\end{aligned}
$$

this clearly indicates that $\dot{\phi}$ is a sum of two contributions.

Before examining each contribution we would like to point out that the chemical potential difference between two superconductors must be the same irrespective of the path it is calculated. Therefore, the following relation is satisfied:

$$
\frac{\partial H_{\text {LIR }}}{\partial N_{L}}-\frac{\partial H_{\text {LIR }}}{\partial N_{R}}=\frac{\partial H_{\text {LBR }}}{\partial N_{L}}-\frac{\partial H_{\text {LBR }}}{\partial N_{R}} .
$$

Let us examine the first contribution in (41). Since the junction is a capacitor in the zeroth approximation, an electric field $\mathbf{E}=-\nabla \varphi$ exists in the insulator. With this electric field $\mathbf{E}$, it is calculated as

$$
\begin{aligned}
\frac{1}{\hbar}\left(\frac{\partial H_{\text {LIR }}}{\partial N_{L}}-\frac{\partial H_{\text {LIR }}}{\partial N_{R}}\right) & =\frac{q}{\hbar}\left(\varphi_{L}-\varphi_{R}\right) \\
& =\int_{L}^{R} q \mathbf{E} \cdot d \mathbf{r}=-\frac{q}{\hbar} W,
\end{aligned}
$$

where $\varphi_{L}$ and $\varphi_{R}$ denotes electrostatic potential of $S_{L}$ and $\mathrm{S}_{R}$, respectively; the integration path connects $\mathrm{S}_{L}$ and $\mathrm{S}_{R}$ via the insulator; $W$ is the voltage across the junction. This contribution is interpreted that it arises form the energy gain of a particle with charge $q$ when it is accelerated in the electric field $\mathbf{E}$ during the particle transfer process from $\mathrm{S}_{L}$ to $\mathrm{S}_{R}$ (it is indicated by the solid-line arrow in Fig. 1). Note that the tunneling through the insulator is a potential barrier tunneling, thus, the energy gain is only by the acceleration in the electric field.

The second contribution in (41) arises from the work done by the battery; it gives rise to the chemical potential difference between $\mathrm{S}_{L}$ and $\mathrm{S}_{R}$, which is given by $-q V$; thus, we have

$\frac{1}{\hbar}\left(\frac{\partial H_{\mathrm{LBR}}}{\partial N_{L}}-\frac{\partial H_{\mathrm{LBR}}}{\partial N_{R}}\right)=\frac{1}{\hbar}\left(\mu_{L}-\mu_{R}\right)=-\frac{q}{\hbar} V$,

where $\mu_{L}$ and $\mu_{R}$ denotes gauge invariant chemical potentials of $\mathrm{S}_{L}$ and $\mathrm{S}_{R}$, respectively. The second process is indicated in Fig. 1 by the dotted-line arrows. Note that the battery can be replaced by some other devices; the important

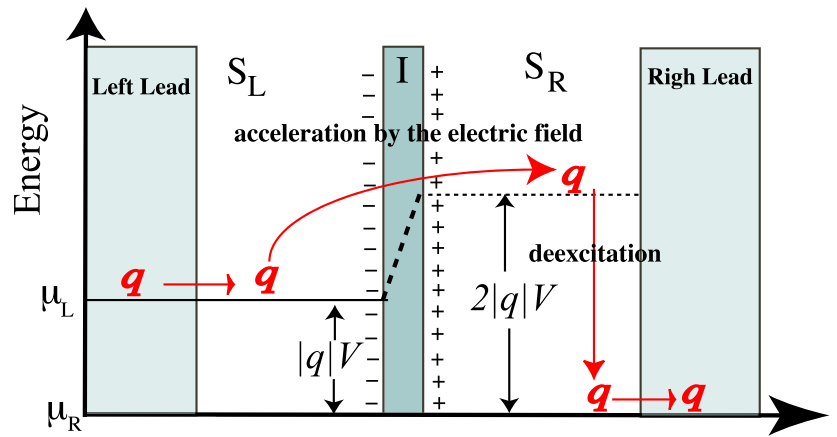

Fig. 2 Energy diagram for the charged particle tunneling through the SIS junction in Fig. 1. It is assumed that $q<0 . \mu_{L}=\partial \bar{H} / \partial N_{L}$ and $\mu_{R}=\partial \bar{H} / \partial N_{R}$ are gauge invariant chemical potentials in $\mathrm{S}_{L}$ and $\mathrm{S}_{R}$, respectively. An electric field is generated in the insulator to maintain the chemical potential difference $\mu_{L}-\mu_{R}=|q| V$. A charge transfer process from the left lead to the right lead is depicted; a charged particle with the energy $\mu_{L}$ enters $S_{L}$ and moves to the weak link made by an insulator $I$. It tunnels through the insulator; thereby, it gains the energy $|q| V$ from the acceleration by the electric field in $I$. By the deexcitation of the energy $2|q| V$, it reaches the level with energy $\mu_{R}$ in $\mathrm{S}_{R}$. Then, it moves toward the right lead, and finally exits from the junction. Application of a radiation field with $\hbar \omega=2|q| V$ will help the deexcitation process

point here is that there is the energy difference of $\mu_{L}$ and $\mu_{R}$ between the particle enters, and that exits.

In the quasi-stationary situation given in (42), Kirchhoff's voltage law is satisfied;

$W=V$.

Over all, the time-derivative of $\phi$ is calculated as

$\dot{\phi}=-\frac{q}{\hbar}(V+W)=-\frac{2 q V}{\hbar}$.

To make the above arguments clearer, let us examine the energetics of the charged particle tunneling. In Fig. 2, the energy diagram for the Josephson junction is depicted. When the tunneling from $S_{L}$ to $S_{R}$ through the insulator occurs, the tunneling particle is accelerated by the electric field inside the insulator, and gains the energy $|q| V$. Another energy gain occurs when a particle enters and another exits from the junction, which is given by $\mu_{L}-\mu_{R}=|q| V$. The total energy gain is, thus, $2|q| V$, which has to be discarded somehow. Applying an electromagnetic field helps this process and doing so will yield the so-called, 'Shapiro-step' in the I-V plot [10].

From (46), the frequency of the AC current is obtained as

$f_{\mathrm{J}}=\frac{2|q| V}{h}$.

The experimentally observed frequency is $2|e| V / h[10]$; therefore, the charge on the particle whose number density is canonical conjugate to $\theta$ is given by

$q=e$ 


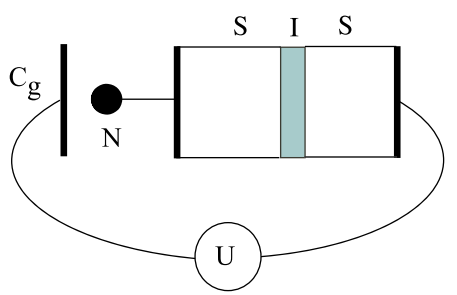

Fig. 3 Set up for the experiment that discriminates whether the charge on the tunneling particle through the Josephson junction is in the unit $e$ or $2 e$. A normal metal quantum dot $\mathrm{N}$ is connected to the Josephson junction SIS. The charging state of $\mathrm{N}$ is controlled by the gate capacitance $\mathrm{C}_{g}$ that is connected to the voltage $U$

This result is surprising since the AC Josephson effect is believed to be due to the Cooper-pair tunneling in the standard theory, thus, the phase $\theta$ is a phase variable that is canonical conjugate to the number density of the Cooper-pairs; in this case $q$ should be $2 e$. If we examine existing derivations so far, it is noticed that the Josephson junction is considered in an open-circuit condition; and the second contribution given in (44) is absent. We believe that the closed-circuit treatment adopted here is relevant to the real experimental situation since the current is measured in one of the leads, not in the junction. It is sensible to include the charge flow between the leads and the junction, thus, the second contribution given in (44) should be included.

The above result upsets the standard theory. Note, however, that it does not mean that pairwise tunnelings do not occur; actually, if the pairing stability is significant, pairwise transfer of particles will be energetically favored. What the present result indicates is that $\theta$ is a conjugate variable to the number density of the charge $e$ particles; thus, the present understanding on the persistent current generation that attributes it to the Cooper-pair flow is not correct. Note also that this statement does not contradict the stabilization by the electron-pair formation and observed asymmetries in the even and odd number of electron systems in some experiments.

For the experimental verification of the present result, we propose an experiment that will discriminate whether the unit of the charge on the tunneling particle is either $e$ or $2 e$. It utilizes a circuit composed of an SIS junction, a normal metal quantum dot, and a gate capacitance connected to the voltage $U$ that controls the charging state of the quantum dot (Fig. 3). The quantum dot and the control-gate are so made that the difference of $e$ or $2 e$-charge change by varying the applied voltage $U$ is detectable. It is also necessary that the two superconductors are not in the size where the asymmetry in even and odd number states is significant. A similar device is constructed and operated for the case where the quantum dot is a superconductor; there, the charging in the unit of $2 e$ is observed due to the stability by the Cooper-pair formation $[32,33]$.
If the charge on the tunneling particle is in the unit $q$ and the Cooper-paring stability is absent, the charge change unit will be $q$ even if $q=e$. By measuring this unit, it will be possible to discriminate whether $q$ is $e$ or $2 e$.

\section{Appearance of $\theta$ from Spin-vortices}

In this section, we will show that the phase variable $\theta$ discussed in the previous section naturally arises from a Berry phase induced by spin-vortices. Actually, its origin is the sign-change caused by the twisting of two basis functions (i.e., the up-spin and down-spin states of an electron) along a loop; this kind of Berry phase was first found by H.C. Longuet-Higgins [34, 35], thus, for definiteness, we may call it the Longuet-Higgins phase.

Let us consider the Hamiltonian given by

$$
\begin{aligned}
\hat{H}= & -\sum_{k, j, \sigma} t_{k j} e^{\frac{i q}{\hbar c} \int_{j}^{k} \mathbf{A} \cdot d \mathbf{r}} c_{k \sigma}^{\dagger} c_{j \sigma} \\
& +\sum_{j, \sigma} q \varphi_{j} c_{j \sigma}^{\dagger} c_{j \sigma}+\hat{H}_{\mathrm{int}}
\end{aligned}
$$

where $j$ denotes the $j$ th site of a crystal lattice and $\sigma$ denotes the electron spin-coordinate. The first term in (49) is the electron hopping Hamiltonian, and $\hat{H}_{\text {int }}$ is the interaction Hamiltonian.

The electromagnetic interaction is introduced by the scalar potential $\varphi$, and the vector potential $\mathbf{A}$, where the latter is included by the Peierls substitution,

$t_{k j} \rightarrow t_{k j} \exp \left(\frac{i q}{\hbar c} \int_{j}^{k} \mathbf{A} \cdot d \mathbf{r}\right)$

When spin-vortices are present the following electron operators $a_{j}$ and $b_{j}$ are convenient since the spin-vortices are included as a prescribed condition as will be seen later. They are related to the original electron annihilation operators $c_{j \uparrow}$ and $c_{j \downarrow}$ as

$$
\left(\begin{array}{c}
a_{j} \\
b_{j}
\end{array}\right)=\frac{e^{i \frac{\chi_{j}}{2}}}{\sqrt{2}}\left(\begin{array}{cc}
e^{i \frac{\xi_{j}}{2}} & e^{-i \frac{\xi_{j}}{2}} \\
-e^{i \frac{\xi_{j}}{2}} & e^{-i \frac{\xi_{j}}{2}}
\end{array}\right)\left(\begin{array}{c}
c_{j \uparrow} \\
c_{j \downarrow}
\end{array}\right) .
$$

In the following, we consider the situation where stable spin-vortices exist; actually, the phase $\xi_{j}$ is regarded essentially as the spin direction in the $x-y$ plane at the $j$ th site [26]. The important point in (51) is that it contains a factor $\exp \left(i \chi_{j} / 2\right)$; the reason for its existence is explained below.

Let us introduce the winding number of $\xi$ around the site $i$ defined as

$w_{i}[\xi]=\frac{1}{2 \pi} \oint_{C_{i}} \nabla \xi \cdot d \mathbf{r}$ 
where $C_{i}$ is a closed path with its center at the $i$ th site. Nonzero $w_{i}$ means that $\xi$ is a multi-valued function.

Now we consider the shift of $\xi$ along a loop that encircles the $i$ th site with assuming that $w_{i}[\xi]=+1$. After the excursion, the value of $\xi$ will be shifted to $\xi+2 \pi$. Then, factors $\exp \left( \pm i \xi_{j} / 2\right)$ in (51) change sign by this phase shift. This is the Longuet-Higgins sign-change due to the twisting of the basis functions. In order to make the transformation matrix single-valued, a phase factor $\exp \left(i \chi_{j} / 2\right)$ must be added; it is so chosen that it compensates for the sign-change of $\exp \left( \pm i \xi_{j} / 2\right)[15-17]$.

The above compensating requirement is satisfied if the sum of two winding numbers

$w_{i}[\xi]+w_{i}[\chi]$

is even, where $w_{i}[\chi]$ is the winding number for $\chi$ defined by

$w_{i}[\chi]=\frac{1}{2 \pi} \oint_{C_{i}} \nabla \chi \cdot d \mathbf{r}$.

Actually, an optimal $\chi$ exists for prescribed winding numbers for $\chi$. We will discuss a way to optimize $\chi$ later.

For the gauge transformation

$\mathbf{A} \rightarrow \mathbf{A}+\nabla f$

electron operators are modified as

$c_{j \sigma} \rightarrow \exp \left(-i \frac{q}{\hbar c} f_{j}\right) c_{j \sigma}$

which, according to (51), means that $\chi$ transforms as

$\chi_{j} \rightarrow \chi_{j}-\frac{2 q}{\hbar c} f_{j}$

Therefore, the two combinations

$-\dot{\chi}+\frac{2 q}{\hbar} \varphi$

and

$\nabla \chi+\frac{2 q}{\hbar c} \mathbf{A}$

are gauge invariant.

Comparing (33)-(34) and (58)-(59), the following relation is suggested:

$\theta=-\frac{\chi}{2}$

as will be seen later, this identification turns out to be affirmative.
Using the new creation and annihilation operators, the hopping term in the Hamiltonian equation (49) is now written as

$$
\begin{aligned}
\hat{K}= & -\sum_{k, j} t_{k j} e^{i \int_{j}^{k}\left(\frac{q}{\hbar c} \mathbf{A}+\frac{\nabla x}{2}\right) \cdot d \mathbf{r}}\left[\cos \frac{\xi_{k}-\xi_{j}}{2}\left(a_{k}^{\dagger} a_{j}+b_{k}^{\dagger} b_{j}\right)\right. \\
& \left.-i \sin \frac{\xi_{k}-\xi_{j}}{2}\left(a_{k}^{\dagger} b_{j}+b_{k}^{\dagger} a_{j}\right)\right]
\end{aligned}
$$

The phase factor $e^{i \int_{j}^{k}\left(\frac{q}{\hbar c} \mathbf{A}+\frac{\nabla \chi}{2}\right) \cdot d \mathbf{r}}$ in $\hat{K}$ indicates that when $\xi$ exhibits non-zero winding numbers, $\nabla \chi$ gives rise to a non-trivial fictitious magnetic field given by

$\mathbf{A}_{\mathrm{fic}}=\frac{\hbar c}{2 q} \nabla \chi$

according to the Peierls substitution.

It has been shown that $\mathbf{A}_{\text {fic }}$ induces loop currents around spin-vortices; and the current density $\mathbf{j}$ is generated by a collection of such loop currents [15-17].

Let us derive equations of motion for $\chi$ by employing the time-dependent variational principle using the following Lagrangian [30]:

$L=\left\langle\Psi\left|\left(i \hbar \frac{\partial}{\partial t}-\hat{H}\right)\right| \Psi\right\rangle$.

The state vector $|\Psi\rangle$ is expressed as

$|\Psi\rangle=\sum_{\alpha} f_{\alpha}|\alpha\rangle$

where $\alpha=\left(n_{a 1}, \ldots, n_{a N_{s}}, n_{b 1}, \ldots, n_{b N_{s}}\right)$ denotes an occupation pattern of electrons; $N_{s}$ is the number of sites, $n_{a j}$ and $n_{b j}$ are eigenvalues of the number operators $a_{j}^{\dagger} a_{j}$ and $b_{j}^{\dagger} b_{j}$ at the $j$ th site, respectively. The state vector $|\alpha\rangle$ is then constructed as

$|\alpha\rangle=\prod_{j \in S_{a}^{\alpha}} a_{j}^{\dagger} \prod_{k \in S_{b}^{\alpha}} b_{k}^{\dagger}|\mathrm{vac}\rangle$

where $S_{a}^{\alpha}$ and $S_{b}^{\alpha}$ denote sets of sites where $n_{a j}$ and $n_{b j}$ are 1 , respectively.

Noting that according to (51), the time-dependences of $a_{j}^{\dagger}$ and $b_{j}^{\dagger}$ arise from the time-dependence of $\chi_{j}$; they are calculated as

$\dot{a}_{j}^{\dagger}=-i \frac{\dot{\chi}_{j}}{2} a_{j}^{\dagger}=-i \frac{\dot{\chi}_{j}}{2} a_{j}^{\dagger} a_{j} a_{j}^{\dagger}$,

$\dot{b}_{j}^{\dagger}=-i \frac{\dot{\chi}_{j}}{2} b_{j}^{\dagger}=-i \frac{\dot{\chi}_{j}}{2} b_{j}^{\dagger} b_{j} b_{j}^{\dagger}$.

Then, the Lagrangian is obtained as

$L=i \hbar \sum_{\alpha} f_{\alpha}^{*} \dot{f}_{\alpha}+\sum_{j} \frac{\hbar}{2} \dot{\chi}_{j} \rho_{j}-\sum_{\alpha, \beta} f_{\alpha}^{*} f_{\beta}\langle\alpha|\hat{H}| \beta\rangle$ 
where $\rho_{j}$ is the number of particles at the $j$ th site given by

$\rho_{j}=\left\langle\Psi\left|a_{j}^{\dagger} a_{j}+b_{j}^{\dagger} b_{j}\right| \Psi\right\rangle$.

From the above Lagrangian, the Hamiltonian is obtained as

$H=\sum_{\alpha, \beta} f_{\alpha}^{*} f_{\beta}\langle\alpha|\hat{H}| \beta\rangle$.

Equations of motion for $f_{\alpha}$ are obtained as

$i \hbar \dot{f}_{\alpha}=\sum_{\beta}\langle\alpha|\hat{H}| \beta\rangle f_{\beta}$

this indicates that the time-dependence of $f_{\alpha}$ describes electronic excitations. In the following, we assume that low energy electronic excitations are eliminated by the effect of $\hat{H}_{\text {int }}$. Then, dynamical variables in the low energy physics are $\rho$ and $\chi$, only.

Let us consider the ground state energy $H_{g}$ for a given $\nabla \xi, \nabla \chi, \mathbf{A}$, and $\varphi$. The Hohenberg-Kohn theorem tells that it is a functional of the density $\rho$ [31], thus, we may express it as

$H_{g}=\bar{H}_{g}\left[\rho, \nabla \chi+\frac{2 q}{\hbar c} \mathbf{A}, \nabla \xi\right]+\sum_{j} q \varphi_{j} \rho_{j}$.

$\nabla \chi$ and $\nabla \xi$ have to be also optimized with constrains of the winding numbers of $\chi$ and $\xi$. We will take $H_{g}$ as a functional of $\chi$ and $\rho$ for a given electromagnetic field.

Using $H_{g}$, the effective Lagrangian for the low energy dynamics is obtained as

$$
\begin{aligned}
L_{\mathrm{eff}} & =\sum_{j} \frac{\hbar}{2} \dot{\chi}_{j} \rho_{j}-\bar{H}_{g}-\sum_{j} q \varphi_{j} \rho_{j}, \\
& =\int d^{3} r \frac{\hbar}{2} \dot{\chi} \rho-\bar{H}_{g}-q \int d^{3} r \varphi \rho .
\end{aligned}
$$

The momentum conjugate to $\chi$ is calculated as

$p_{\chi}=\frac{\delta L_{\mathrm{eff}}}{\delta \dot{\chi}}=\frac{\hbar}{2} \rho$,

thus, $\rho$ and $-\frac{1}{2} \chi$ are conjugate variables in accordance with the previous suggestion.

Finally, the equations of motion that correspond to (30) and (31) are obtained;

$\dot{\rho}=-\frac{2}{\hbar} \frac{\delta \bar{H}_{g}}{\delta \chi}$

and

$\dot{\chi}-\frac{2 q}{\hbar} \varphi=\frac{2}{\hbar} \frac{\delta \bar{H}_{g}}{\delta \rho}$.
Let us consider the Josephson junction problem; for the Josephson junction case, the Hamiltonian is decomposed as

$\hat{H}_{\text {Josephson }}=\hat{K}_{\text {Link }}+\hat{H}_{\text {Rest }}$

where the hopping term through the weak link $\hat{K}_{\text {Link }}$ is given by

$$
\begin{aligned}
\hat{K}_{\text {Link }}= & -\sum_{k \in \mathrm{S}_{L}, j \in \mathrm{S}_{R}} T_{k j} \exp \left(i \int_{j}^{k} \frac{q}{\hbar c} \mathbf{A} \cdot d \mathbf{r}\right. \\
& \left.+i \frac{\chi_{k}+\chi_{L}-\chi_{j}-\chi_{R}}{2}\right) \\
& \times\left[\cos \frac{\xi_{k}-\xi_{j}}{2}\left(a_{k}^{\dagger} a_{j}+b_{k}^{\dagger} b_{j}\right)\right. \\
& \left.-i \sin \frac{\xi_{k}-\xi_{j}}{2}\left(a_{k}^{\dagger} b_{j}+b_{k}^{\dagger} a_{j}\right)\right]+ \text { h.c. } ;
\end{aligned}
$$

$T_{k j}$ is the hopping parameter across the insulator, $\chi_{L}$ and $\chi_{R}$ correspond to $-2 \theta_{L}$ and $-2 \theta_{R}$ in (10) and (11), respectively, and represent parts of $\chi$ that are indeterminate by the optimization of $\nabla \chi$ in each superconductor. $\hat{H}_{\text {Rest }}$ simply describes the rest of the Hamiltonian.

Using $\hat{K}_{\text {Link }}$, the electric current through the weak link is expressed as

$$
\begin{aligned}
J= & \sum_{k \in \mathrm{S}_{L}, j \in \mathrm{S}_{R}} J_{k j} \sin \left(\frac{\chi_{R}+\chi_{j}-\chi_{L}-\chi_{k}}{2}\right. \\
& \left.-\int_{j}^{k} \frac{q}{\hbar c} \mathbf{A} \cdot d \mathbf{r}-\alpha_{k j}\right)
\end{aligned}
$$

where

$J_{k j}=\frac{2 q T_{k j} A_{k j}}{\hbar} ;$

$A_{k j}$ and $\alpha_{k j}$ are the amplitude and phase of the following expectation value:

$$
\begin{aligned}
A_{k j} e^{i \alpha_{k j}}= & \left\langle\cos \frac{\xi_{k}-\xi_{j}}{2}\left(a_{k}^{\dagger} a_{j}+b_{k}^{\dagger} b_{j}\right)\right. \\
& \left.-i \sin \frac{\xi_{k}-\xi_{j}}{2}\left(a_{k}^{\dagger} b_{j}+b_{k}^{\dagger} a_{j}\right)\right\rangle
\end{aligned}
$$

We may use $\alpha_{k j}$ and $A_{k j}$ that are calculated for the $\chi_{j}-\chi_{k}=0$ and $\mathbf{A}=0$ case for other values for $\chi_{R}-\chi_{L}$ and $\mathbf{A}$. Under this approximation, the electric current in (78) becomes a functional of $\chi_{R}-\chi_{L}$ for a given $\mathbf{A}$. The timedependences of $\chi_{R}$ and $\chi_{L}$ are obtained from (13) and (15) substituting $H_{g}$ to $H$, and $\chi$ to $-2 \theta$. If $k, j$-dependences are neglected, the current in (78) reduces to that in (37). 


\section{The Appearance of Stable $\nabla \chi$ States}

Now we discuss $\hat{H}_{\text {int }}$ that will give rise to the required stability in $\nabla \chi$. We consider a two-dimensional conduction plane and take the following interacting Hamiltonian,

$$
\hat{H}_{\mathrm{int}}=U \sum_{j} a_{j}^{\dagger} a_{j} b_{j}^{\dagger} b_{j}+\hat{H}^{\prime}
$$

and impose the following conditions that lead the system to be in the effectively half-filled Mott insulator (EHFMI) state [15, 26]: (1) the parameter $U$ satisfies the strong correlation condition, $U \gg\left|t_{j k}\right|$; (2) doped carriers in the half-filled parent compound are assumed to become small polarons with very small mobility (this is assumed to be achieved by the carrier-lattice interaction contained in $\hat{H}^{\prime}$ ); (3) spinvortices are formed with doped carriers at their centers (the interaction that stabilizes spin-vortices are assumed to be contained in $\hat{H}^{\prime}$ ).

In order to obtain the electronic states for the EHFMI state, we neglect the small polaron hopping, and employ the following reduced Hamiltonian;

$\hat{H}_{\mathrm{EHFMI}}=\hat{K}+U \sum_{j \in \text { acc. sites }} a_{j}^{\dagger} a_{j} b_{j}^{\dagger} b_{j}$,

where only accessible sites are included (or small polaron occupied sites are excluded) in $\hat{K}$ and the Coulomb term.

In the following we consider situations where the magnetic field effect is negligible, thus, $\mathbf{A}=0$ can be used in $\hat{K}$.

We employ the following mean field Hamiltonian to obtain an approximate ground state:

$$
\bar{H}_{\mathrm{EHFMI}}=\hat{K}+U \sum_{j \in \text { acc. sites }}\left(\left\langle a_{j}^{\dagger} a_{j}\right\rangle b_{j}^{\dagger} b_{j}+\left\langle b_{j}^{\dagger} b_{j}\right\rangle a_{j}^{\dagger} a_{j}\right) .
$$

In the effectively half-filled situation, the obtained ground state is a Slater determinant of single-particle wave functions for a filled lower band. In this respect we will call this EHFMI state as the effectively half-filled Slater state (EHFSS). The mean field calculation also yields singleparticle wave functions for an empty band which exists above the lower band with an energy gap of about $U$. One might think that the ground state is a band insulator. However, it is not; it can be a current carrying state if spinvortices exist thanks to the fictitious magnetic $\left(\mathbf{A}_{\text {fic }}\right.$ in (62)) induced by them.

In the EHFMI state, we have the following situation;

$\left\langle a_{j}^{\dagger} a_{j}+b_{j}^{\dagger} b_{j}\right\rangle=1$

where $j$ denotes the accessible sites. Since the Slater determinant for the filled band can be also described by a localized basis, the on-site Coulomb energy is small; thus, we can obtain current carrying states with small Coulomb energy. The current is intact as long as the spin-vortices are intact. Since the spin-vortices are expected to be stable, these current carrying states will be also stable.

First, we consider the optimization of $\xi$ appears in (51). Actually, for a given $\xi$ and $\chi$, low energy spin-wave excitations from the ground state are possible; thus, we will optimize $\xi$ using a spin Hamiltonian.

Let us construct the spin Hamiltonian for the EHFMI. For that purpose, we adopt the condition

$a_{j}^{\dagger} a_{j}+b_{j}^{\dagger} b_{j}=1$

as a substitute of (84) since this makes calculations much easier.

Taking the on-site Coulomb interaction term as the zeroth Hamiltonian and $\hat{K}$ as a perturbation, the spin Hamiltonian is obtained as [26]

$$
\begin{aligned}
\hat{H}_{\text {spin }}= & \frac{1}{U} \sum_{k, j} t_{j k}^{2} \sin ^{2} \frac{\xi_{j}-\xi_{k}}{2}\left(\tilde{S}_{k}^{-} \tilde{S}_{j}^{-}+\tilde{S}_{k}^{+} \tilde{S}_{j}^{+}\right. \\
& \left.-2 \tilde{S}_{k}^{Z} \tilde{S}_{j}^{Z}-\frac{1}{2}\right)+\frac{1}{U} \sum_{k, j} t_{j k}^{2} \cos ^{2} \frac{\xi_{j}-\xi_{k}}{2} \\
& \times\left(\tilde{S}_{k}^{-} \tilde{S}_{j}^{+}+\tilde{S}_{k}^{+} \tilde{S}_{j}^{-}+2 \tilde{S}_{k}^{Z} \tilde{S}_{j}^{Z}-\frac{1}{2}\right),
\end{aligned}
$$

where spin operators, $\tilde{S}_{j}^{+}, \tilde{S}_{j}^{-}$, and $\tilde{S}_{j}^{Z}$ are defined as

$\tilde{S}_{j}^{+}=b_{j}^{\dagger} a_{j}$,

$\tilde{S}_{j}^{-}=a_{j}^{\dagger} b_{j}$,

$\tilde{S}_{j}^{Z}=\frac{1}{2}\left(b_{j}^{\dagger} b_{j}-a_{j}^{\dagger} a_{j}\right)$.

In the optimization, we simply use the zeroth order ground state given by

$$
|0\rangle=\prod_{i \in \text { acc. sites }} a_{i}^{\dagger}|\mathrm{vac}\rangle,
$$

where $|v a c\rangle$ is the vacuum state.

Then, we optimize $\nabla \xi$ by minimizing the following energy

$\left\langle 0\left|\hat{H}_{\text {spin }}\right| 0\right\rangle=-\frac{1}{U} \sum_{k, j} t_{j k}^{2} \sin ^{2} \frac{\xi_{j}-\xi_{k}}{2}$

with prescribed winding numbers around small polaron occupied sites.

Next, we optimize $\nabla \chi$. In order to obtain the optimized $\nabla \chi$, we use the mean field Hamiltonian in (83). In this case, the ground state is described by a Slater determinant, thus, we treat the EHFMI state as the EHFSS. 
In the optimizing process, careful treatment of the multivalued phase factor

$\exp \left(\frac{i}{2} \int_{j}^{k} \nabla \chi \cdot d \mathbf{r}\right)$

is necessary. If we simply employ the basis $\left\{a_{j}^{\dagger}|\mathrm{vac}\rangle\right.$, $\left.b_{j}^{\dagger}|\mathrm{vac}\rangle\right\}_{j \in \text { acc. sites, }}$ a digital computer will treat the above phase factor as single-valued functions; then, the Berry phase effect disappears. In order to overcome this problem, we prepare the basis with required multi-valuedness in the following manner. This is a tight-binding model variant of the method employed to deal with the Longuet-Higgins sign-change in molecular problems [36-38].

We first solve the fictitious problem, i.e., we consider a system with spin-vortices but with $\chi=0$; here, we utilize particle operators

$\tilde{a}_{j}=e^{-i \frac{1}{2} \chi_{j}} a_{j}$,

$\tilde{b}_{j}=e^{-i \frac{1}{2} \chi_{j}} b_{j}$,

instead of $a_{j}$ and $b_{j}$ as a basis.

In the calculation of the Hamiltonian matrix elements, the phase difference $\xi_{k}-\xi_{j}$ in the Hamiltonian should be chosen in the range

$-\pi<\xi_{k}-\xi_{j} \leq \pi$.

After the diagonalization of the above-constructed Hamiltonian matrix, the following single-particle states are obtained:

$|\tilde{\alpha}\rangle=\sum_{j}\left(A_{j}^{\alpha} \tilde{a}_{j}^{\dagger}+B_{j}^{\alpha} \tilde{b}_{j}^{\dagger}\right)|\mathrm{vac}\rangle$,

where $A_{j}^{\alpha}$ and $B_{j}^{\alpha}$ are numerical values obtained from the diagonalization. In this way we obtain, $2 N_{e}$ linearly independent $|\tilde{\alpha}\rangle$, where $N_{e}$ is the number of electrons; we include the nonlocal effect arising from the Berry phase in these extended states.

Next we prepare $2 N_{e}$ basis functions that satisfies the required boundary condition from $\{|\tilde{\alpha}\rangle\}_{1}^{2 N_{e}}$ as

$|\alpha\rangle=\sum_{j}\left(A_{j}^{\alpha} a_{j}^{\dagger}+B_{j}^{\alpha} b_{j}^{\dagger}\right)|\mathrm{vac}\rangle$.

The Hamiltonian matrix that includes the Berry phase effect is constructed using $\{|\alpha\rangle\}_{1}^{2 N_{e}}$ as a basis. Note that, in the calculation of the matrix elements, the phase difference $\chi_{k}-\chi_{j}$ should be chosen in the range

$-\pi<\chi_{k}-\chi_{j} \leq \pi$
By numerically diagonalizing the Hamiltonian matrix constructed with the basis $\{|\alpha\rangle\}_{1}^{2 N_{e}}$, single-particle state vectors

$|I\rangle=\sum_{\alpha} C_{I}^{\alpha}|\alpha\rangle$

are obtained. The ground state is obtained as a Slater determinant of the lowest $N_{e}$ energy functions from $\{|I\rangle\}_{1}^{2 N_{e}}$. Finally, $\nabla \chi$ is optimized by minimizing the energy calculated with the above-obtained Slater determinant.

Strictly speaking, the optimization of $\xi$, that of $\chi$, and the construction of the Slater-determinant ground state from the mean field solution have to be self-consistently performed; however, the sequential optimization described above is simple and will be adequate for many purposes.

By changing the winding numbers for $\chi$, states with different current flow patterns are obtained [15-17]. They are not so much different in energy, thus, when an external magnetic field is applied, the ground state will be chosen from them in a very flexible manner.

When an external magnetic field is applied, an approximate ground state may be obtained by simply optimizing $\nabla \chi$ in the presence of $\mathbf{A}$. The optimal state will be a state with a minimal current carrying state; thus, the system will try to exclude the magnetic field by minimizing the current flow inside the bulk; such an exclusion of a magnetic field is nothing but the Meissner effect [17].

The condition for the optimal $\chi$ will be given by

$\int_{j}^{k}\left(\frac{q}{\hbar c} \mathbf{A}+\frac{\nabla \chi}{2}\right) \cdot d \mathbf{r}=$ const.

in deep inside a specimen, which will be obtained by zerocurrent in the bulk from the hopping term in (61).

The above condition indicates that if the specimen is a ring-shaped, the quantization of magnetic flux is realized; by taking a closed path that encircles the hole of the ring, the above condition yields

$\oint_{\text {loop }}\left(\frac{q}{\hbar c} \mathbf{A}+\frac{\nabla \chi}{2}\right) \cdot d \mathbf{r}=0$

This is rewritten as

$\oint_{\text {loop }} \mathbf{A} \cdot d \mathbf{r}=-\frac{\hbar c}{2 q} \oint_{\text {loop }} d \chi$.

Since $\chi$ is a function of period $2 \pi$, this will give the flux quantization in the unit $\Phi_{0}$ with $q=e$.

\section{Macroscopic Quantum Interference}

The oscillation of the maximum zero-voltage current with varying an external magnetic field is observed in a circuit 
Fig. 4 Sketch of a SQUID structure and plot of the maximum current through the SQUID structure, $J_{\max }^{e}$, vs the magnetic flux through the ring, $\Phi_{\text {ring }} . \Phi_{\text {junc }}$ is chosen to be $\Phi_{\text {junc }}=0.1 \Phi_{\text {ring }}$

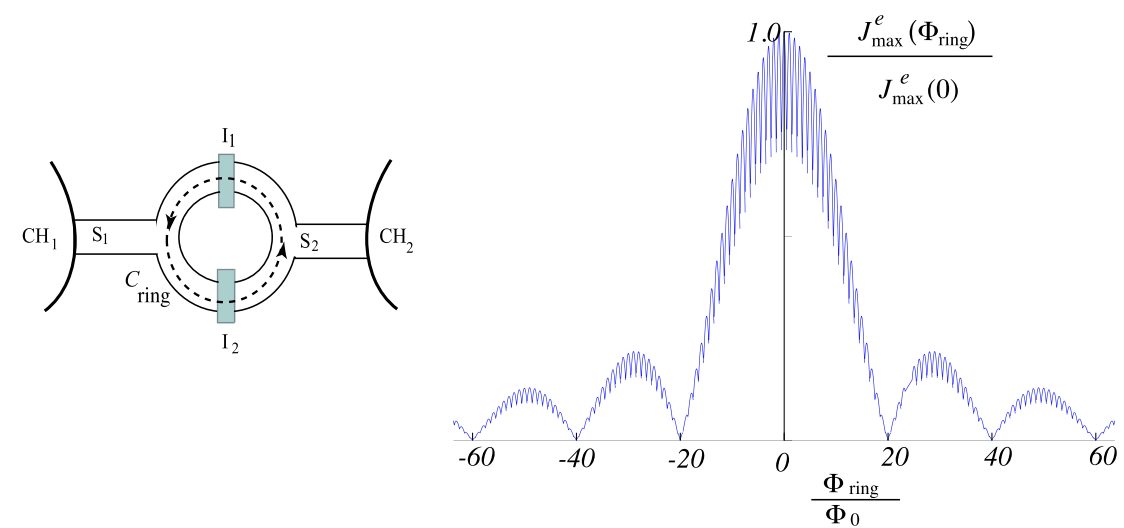

with two SIS junctions (the SQUID structure) shown in Fig. 4. This oscillation is a macroscopic quantum interference phenomenon brought about by the phase $\theta$. In the standard theory, this phenomenon is explained using $\theta$ that is conjugate to the number density of $2 e$ charge carriers. In this section, however, we explain it using the $\theta$ that is conjugate to the number density of $e$ charge carriers discussed in previous sections. The key ingredient is the winding number of $\chi$.

Let us calculate the current through a SQUID structure in Fig. 4. The SQUID structure is made of the two SIS junctions that are assumed to be identical. The total current is a sum of currents through the two junctions. If the magnetic flux through each junction area is neglected, the total current is calculated as

$J=J_{0} \sin \phi_{c}+J_{0} \sin \left(\phi_{c}-\frac{\pi \Phi_{\text {ring }}}{\Phi_{0}}+w_{\text {ring }} \pi\right)$,

where $\Phi_{\text {ring }}$ is the flux through the ring region given by

$\Phi_{\text {ring }}=\oint_{\mathrm{C}_{\text {ring }}} \mathbf{A} \cdot d \mathbf{r}$

and $w_{\text {ring }}$ is the winding number of $\chi$ along $\mathrm{C}_{\text {ring }}$ given by

$w_{\text {ring }}=\frac{1}{2 \pi} \oint_{\mathrm{C}_{\text {ring }}} \nabla \chi \cdot d \mathbf{r}$.

The current in (103) exhibits an interference effect; the phase difference for the interference is composed of two contributions: one is the contribution from the AharonovBohm phase from the magnetic filed penetrating through the ring given by

$-\pi \frac{\Phi_{\text {ring }}}{\Phi_{0}}$

the other is the contribution from the winding number of $\chi$ given by

$w_{\text {ring }} \pi$.
In the standard $2 e$ charge case, the former is given by $-2 \pi \Phi_{\text {ring }} / \Phi_{0}$, and the latter is a multiple of $2 \pi$ which can be taken away because it is a period of the sine function.

The maximum current depends on whether $w_{\text {ring }}$ is even or odd. If $w_{\text {ring }}$ is even, it is calculated as

$$
\begin{aligned}
& J_{\max }^{\text {even }}\left(\Phi_{\text {junc }}, \Phi_{\text {ring }}\right) \\
& =J_{\max }^{\text {even }}(0,0)\left|\frac{\sin \left(\frac{\pi \Phi_{\text {junc }}}{2 \Phi_{0}}\right)}{\frac{\pi \Phi_{\text {junc }}}{2 \Phi_{0}}}\right|\left|\cos \left(\frac{\pi \Phi_{\text {ring }}}{2 \Phi_{0}}\right)\right|,
\end{aligned}
$$

and if it is odd, it is given by

$$
\begin{aligned}
& J_{\text {max }}^{\text {odd }}\left(\Phi_{\text {junc }}, \Phi_{\text {ring }}\right) \\
& =J_{\max }^{\text {even }}(0,0)\left|\frac{\sin \left(\frac{\pi \Phi_{\text {junc }}}{2 \Phi_{0}}\right)}{\frac{\pi \Phi_{\text {junc }}}{2 \Phi_{0}}}\right|\left|\sin \left(\frac{\pi \Phi_{\text {ring }}}{2 \Phi_{0}}\right)\right|,
\end{aligned}
$$

where the effect of the flux through each junction region $\Phi_{\text {junc }}$ is included.

If we take into account the change of $w_{\text {ring }}$, the maximum current $J_{\max }^{e}$ is the largest of $J_{\max }^{\text {even }}$ and $J_{\max }^{\text {odd }}$ defined by

$J_{\max }^{e}=\operatorname{Max}\left\{J_{\max }^{\text {even }}, J_{\max }^{\text {odd }}\right\}$.

In Fig. 4, a plot of $J_{\max }^{e}$ as a function of $\Phi_{\text {ring }}$ is depicted. It exhibits a profile similar to the one observed in experiments [28], showing peaks separated by $\Phi_{0}$.

The corresponding maximum current from the $2 e$ charge carrier theory is given by

$$
\begin{aligned}
& J_{\max }^{2 e}\left(\Phi_{\text {junc }}, \Phi_{\text {ring }}\right) \\
& =J_{\max }^{2 e}(0,0)\left|\frac{\sin \left(\frac{\pi \Phi_{\text {junc }}}{\Phi_{0}}\right)}{\frac{\pi \Phi_{\text {junc }}}{\Phi_{0}}}\right|\left|\cos \left(\frac{\pi \Phi_{\text {ring }}}{\Phi_{0}}\right)\right| .
\end{aligned}
$$

A noticeable difference is that while $J_{\max }^{2 e}$ is zero at $\frac{\Phi_{\text {ring }}}{\Phi_{0}}=\frac{1}{2}+n$ 
Fig. 5 a Plot of $J_{\max }^{\text {even }}$ as a

function of $\Phi_{\text {ring. It shows a }}$

"0-SQUID (or edge-junction)"

pattern. b Plot of $J_{\max }^{\text {odd }}$ as a

function of $\Phi_{\text {ring }}$ it shows a

" $\pi$-SQUID (or

corner-junction)" pattern.

$\Phi_{\text {junc }}=0.5 \Phi_{\text {ring }}$
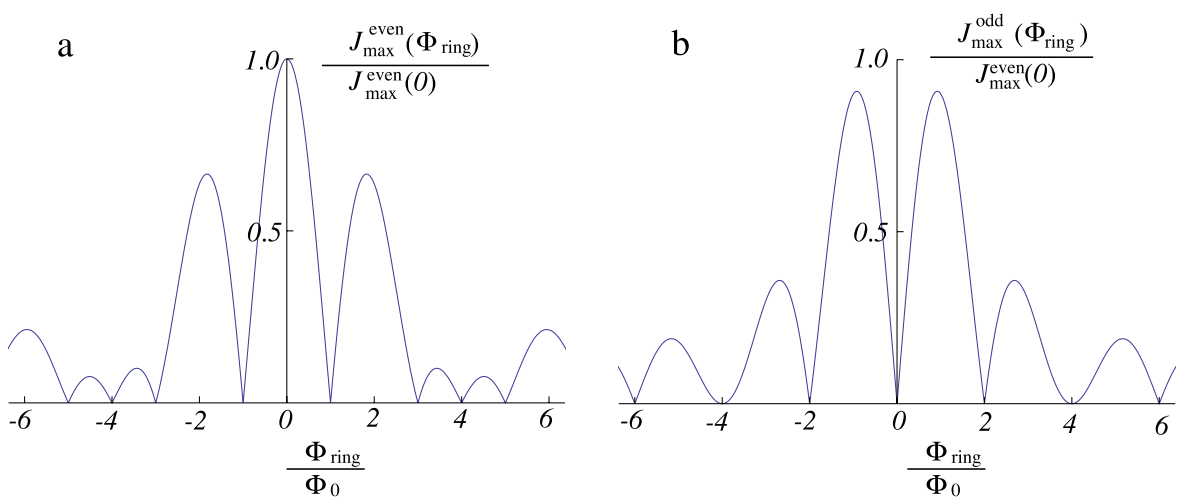

where $n$ is an integer, it is not zero for $J_{\max }^{e}$. This absence of the zero current points may be used to discriminate the spinvortex superconductivity from the standard one. Actually, the absence was observed in the experiment, but the reason for it has been attributed to a background current from various effects.

As shown in Fig. 5, if even $w_{\text {ring }}$ and odd $w_{\text {ring }}$ cases are separately realized, the so-called " 0 -SQUID (or edgejunction)" pattern and the " $\pi$-SQUID (or corner-junction)" pattern are obtained, respectively [29]. The appearance of these patterns are currently attributed to the d-wave symmetry of the order parameter. However, we have shown that it can be explained due to the separated appearance of the even and odd $w_{\text {ring }}$ cases using the present theory.

\section{Concluding Remarks}

We have presented a theory of superconductivity based on the persistent current generation by a Berry phase due to the spin-vortex formation. It attributes the phase $\theta$ to the Berry phase arising from the stable (or quasi-stable) spin-vortex formation. We have found that the observed AC Josephson frequency actually indicates that the charge on the tunneling particle is in the unit $e$, not $2 e$ if the junction is treated as a part of a closed-circuit with including the contribution from flow-in and -out of particles from the leads. Since the experiment is done in such a closed-circuit situation, it is sensible to include the flow-in and -out. For the verification of our claim, we have proposed an experiment that discriminates whether the carrier charge unit is $e$ or $2 e$.

If our claim turns out to be correct, the Berry phase origin of the phase $\theta$ is the only one left that explains the observed flux quantization unit $\Phi_{0}$ and the AC Josephson frequency $f_{\mathrm{J}}$ with $q=e$. This also indicates that the BCS theory is incomplete as to the origin of supercurrent, thus, cannot predict whether a particular compound is superconductor or not. It is well-known that the BCS theory gives an excellent estimate of $T_{\mathrm{c}}$ and properties associated with the energy gap formation at $T_{\mathrm{c}}$. Therefore, there must be a strong relation between the Cooper-pair condensation and the onset of the superconducting state. There are also plenty of experiments that indicate asymmetries in the even and odd number of electron systems, which can be attributed to the electron-pairing stabilization.

The reason for the fact that the BCS theory gives an excellent estimate of $T_{\mathrm{c}}$ may be the following: As is argued in Sect. 3, in order that the system is in the superconducting state, low energy electronic excitations that destabilize $\nabla \theta$ must be absent. The BCS theory provides the mechanism in which this absence occurs by the energy gap formation. Our theory assumes that the system is in the EHFMI state, where localized polarons are formed as stabilizing centers of spin-vortices. In general, the localization occurs more easily in the bulk than in the surface region since the shielding of charge and chemical bond formation are easier in the bulk than in the surface region. Then, electronic excitations in the surface region will be the last low energy excitations to be eliminated for the stability of $\nabla \theta$. If this elimination is brought about by the energy gap formation by the Cooperpair condensation, the BCS theory will give an excellent estimate of $T_{\mathrm{c}}$.

It is also worth noting that a statistical analysis reveals that the occurrence of superconductivity is strongly correlated to the fact that carriers are holes [20]. Since holes are known to become small polarons more frequently than electrons, the above-mentioned situation seems to fit the statistical analysis.

The above explanation is, however, not applicable for superconductors in general since all of them are not categorized as strongly correlated systems. For materials with weak correlations, some other mechanism is necessary for the spin-vortex formation. One possible mechanism is that the energy gap formation by the Cooper-pair condensation induces a band reconstruction that creates a situation with $U \gg\left|t_{i j}\right|$ in the relevant band. It is also possible that impurities or defects become stabilizing centers of spin-vortices, and the Cooper-pairing-gap provides the final stabilization for the induced loop currents. In this respect, it is worth noting that recent optical spectroscopy in $\mathrm{Pb}$ observed the 
emergence of peaks in the superconducting state; they seem to originate from phonon-assisted processes, and are not explainable within the standard BCS approach [39].

Let us consider superconductivity in cuprates. In the cuprate superconductors, experiments that measure electronic states in the surface region such as ARPES and STS see rather itinerant electrons; however, experiments probe the bulk see localized-carrier effects. Neutron scattering experiments observe spin-wave excitations that appear to arise from local moments [40-42]. Actually, the observed spinwave excitations are well-explained by assuming that the system is in the EHFMI state [26]. Furthermore, the EXAFS detects bond-length fluctuations that are correlated with the occurrence of superconductivity [43]; and this fluctuation is explained by the small polaron formation [44]. Thus, the above-mentioned situation, "the EHFMI state in the bulk, and the BCS-type gap formation in the surface region", seems to be relevant to the cuprates.

We have a view that spin-vortex-induced loop currents exist below the pseudogap temperature $T^{*}$ and become supercurrent below the superconducting transition temperature $T_{\mathrm{c}}$. This view naturally explains the change of the sign of the Hall coefficient from positive to negative as the temperature is lowered [45]; the positive sign indicates that the dominant charge carriers are doped-holes, and the negative that they are electrons in the loop currents.

An experiment shows that the BCS-type gap formation does not necessary lead the system to a superconducting state. It is seen in $\mathrm{La}_{1-x} \mathrm{Ba}_{x} \mathrm{CuO}_{4}$, superconductivity is anomalously suppressed at $x=1 / 8$ [21]. Although the pairing-gap exists at this doping level as in the near-by doping levels, somehow the system fails to be superconducting. Actually, this anomaly may be explained in the spin-vortex superconductivity theory; if spin-vortices line up to form a stripe order, the loop currents that flow along the both sides of the stripe cancel to be zero since they are opposite in the direction and same in the magnitude.

The present work suggests the reason why the standard theory of superconductivity does not have a predicting power for the occurrence of superconductivity in a particular compound; it may be due to the fact that its attribution of the origin of the phase $\theta$ is wrong. A new theory of superconductivity may emerge by identifying $\theta$ as the Berry phase (or the Longuet-Higgins phase) from spin-vortices. It contains the BCS pairing mechanism as a part for the mechanism that stabilize $\theta$. It does not violate the superselection rule for the total charge, and, thus, enable us to calculate persistent current related phenomena in an atomistic way without recourse to the Ginzburg-Landau phenomenological equation.

Open Access This article is distributed under the terms of the Creative Commons Attribution Noncommercial License which permits any noncommercial use, distribution, and reproduction in any medium, provided the original author(s) and source are credited.

\section{References}

1. Onnes, H.K.: Leiden Commun. 120b, 122b, 124c (1911)

2. Bardeen, J., Cooper, L.N., Schrieffer, J.R.: Phys. Rev. 108, 1175 (1957)

3. London, F.: Nature 141, 543 (1938)

4. Penrose, O., Onsager, L.: Phys. Rev. 104, 576 (1956)

5. Ginzburg, V.L., Landau, L.D.: Zh. Eksp. Theor. Fiz. 20, 1064 (1950)

6. Anderson, P.W.: Rev. Mod. Phys. 38, 298 (1966)

7. Josephson, B.D.: Phys. Lett. 1, 251 (1962)

8. Anderson, P.W., Rowell, J.M.: Phys. Rev. Lett. 10, 230 (1963)

9. Deaver, B.S. Jr., Fairbank, W.M.: Phys. Rev. Lett. 7, 43 (1961)

10. Shapiro, S.: Phys. Rev. Lett. 11, 80 (1963)

11. Leggett, A.J.: Rev. Mod. Phys. 73, 307 (2001)

12. Wick, G.C., Wightman, A.S., Wigner, P.E.: Phys. Rev. 88, 101 (1952)

13. Peierls, R.: J. Phys. A, Math. Gen. 24, 5273 (1991)

14. Leggett, A.J.: Quantum Liquids. Oxford Univ. Press, Oxford (2006)

15. Koizumi, H.: J. Phys. Soc. Jpn. 77, 034712 (2008)

16. Koizumi, H.: J. Phys. Chem. A 113, 3997 (2009)

17. Koizumi, H.: J. Phys. A 43, 354009 (2010)

18. London, F.: Superfluids, vol. 1. Dover, New York (1961)

19. Anderson, P.W.: Phys. Rev. 110, 827 (1958)

20. Hirsch, J.E.: Phys. Rev. B 55, 9007 (1997)

21. He, R.-H., Tanaka, K., Mo, K.-S., Sasagawa, T., Fujita, M., Adachi, T., Mannella, N., Yamada, K., Koike, Y., Hussain, Z., Shen, Z.-X.: Nat. Phys. 5, 119 (2009)

22. Xia, J., Schemm, E., Deutscher, G., Kivelson, S.A., Bonn, D.A., Hardy, W.H., Liang, R., Siemons, W., Koster, G., Fejer, M.M., Kapitulnik, A.: Phys. Rev. Lett. 100, 127002 (2008)

23. Kapitulnik, A., Xia, J., Schemm, E., Palevski, A.: New J. Phys. 11, 055060 (2009)

24. Li, Y., Balédent, V., Yu, G., Barišić, N., Hradil, K., Mole, R.A., Sidis, Y., Steffens, P., Zhao, X., Bourges, P., Greven, M.: Nature 468, 283 (2010)

25. Anderson, P.W.: Basic Notions of Condensed Matter Physics. Benjamin/Cummings, Menlo Park (1984)

26. Koizumi, H.: J. Phys. Soc. Jpn. 77, 104704 (2008)

27. Slater, J.C.: Phys. Rev. 82, 538 (1951)

28. Jaklevic, R.C., Lambe, J., Mercereau, J.E., Silver, A.H.: Phys. Rev. 140, A1628 (1965)

29. Wollman, D.A., van Harlingen, D.J., Giapintzakis, J., Ginsberg, D.M.: Phys. Rev. Lett. 74, 797 (1995)

30. Kerman, A.K., Koonin, S.E.: Ann. Phys. 100, 332 (1976)

31. Hohenberg, P., Kohn, W.: Phys. Rev. 136, B864 (1964)

32. Bouchiat, V., Vion, D., Joyez, P., Esteve, D., Devoret, M.H.: Phys. Scr. T 76, 165 (1998)

33. Vion, D., Aassime, A., Cottet, A., Joyez, P., Pothier, H., Urbina, C., Esteve, D., Devoret, M.H.: Science 296, 886 (2002)

34. Longuet-Higgins, H.C., Öpik, U., Pryce, M.H.L., Sack, R.A.: Proc. R. Soc. Lond. Ser. A, Math. Phys. Sci. 244, 1 (1958)

35. Herzberg, G., Longuet-Higgins, H.C.: Discuss. Faraday Soc. 35, 77 (1963)

36. O'Brien, M.C.M.: Proc. R. Soc. Lond. Ser. A, Math. Phys. Sci. 281, 323 (1964)

37. Wu, Y.-S.M., Kupperman, A., Lepetit, B.: Chem. Phys. Lett. 186, 319 (1991)

38. von Busch, H., Dev, V., Eckel, H.-A., Kasahara, S., Wang, J., Demtröder, W., Sebald, P., Meyer, W.: Phys. Rev. Lett. 81, 4584 (1998)

39. Mori, T., Nicol, E.J., Shiizuka, S., Kuniyasu, K., Nojima, T., Toyota, N., Carbotte, J.P.: Phys. Rev. B 77, 174515 (2008) 
40. Hayden, S.M., Mook, H.A., Dai, P., Perring, T.G., Doğan, F.: Nature 429, 531 (2004)

41. Tranquada, J.M., Woo, H., Perring, T.G., Goka, H., Gu, G.D., Xu, G., Fujita, M., Yamada, K.: Nature 429, 534 (2004)

42. Xu, G., Gu, G.D., Hücker, M., Fauqué, B., Perring, T.G., Regnault, L.P., Tranquada, J.M.: Nat. Phys. 5, 642 (2009)

43. Zhang, C.J., Oyanagi, H.: Phys. Rev. B 79, 094521 (2009)
44. Miyaki, S., Makoshi, K., Koizumi, H.: J. Phys. Soc. Jpn. 77, 034702 (2008)

45. LeBoeuf, D., Doiron-Leyraud, N., Levallois, J., Daou, R., Bonnemaison, J.-B., Hussey, N.E., Balicas, L., Ramshaw, B.J., Liang, R., Bonn, D.A., Hardy, W.N., Adachi, S., Proust, C., Taillerfer, L.: Nature 450, 533 (2007) 